\author{
Pure Mathematical Sciences, Vol. 3, 2014, no. 3, 105 - 112 \\ HIKARI Ltd, www.m-hikari.com \\ http://dx.doi.org/10.12988/pms.2014.436
}

\title{
A Radial Distribution on the Heisenberg Group
}

\author{
M. E. Egwe \\ Department of Mathematics \\ Universty of Ibadan \\ Ibadan 200005, Oyo State, Nigeria
}

Copyright (C) 2014 M. E. Egwe. This is an open access article distributed under the Creative Commons Attribution License, which permits unrestricted use, distribution, and reproduction in any medium, provided the original work is properly cited.

\begin{abstract}
Let $\mathbb{H}_{n}$ denote the $(2 \mathrm{n}+1)$-dimensional Heisenberg group and let $K$ be a compact subgroup of $A u t\left(\mathbb{H}_{n}\right)$, the group of automorphisms of $\mathbb{H}_{n}$. In this paper, a spherical radial function, $\Phi(h)$ on the Heisenberg group with respect to $K$ is raised and studied. It is then shown that $\Phi(h)$ is positive definite and compactly supported in the space of distributions on the group.
\end{abstract}

Mathematics Subject Classification: 222E45, 22E46, 46E10, 46E35, $46 \mathrm{~T} 30$

Keywords: Heisenberg group, Radial functions, distributions, compact support, spherical function

\section{Preliminaries}

The Heisenberg group (of order $n$ ), $\mathbb{H}_{n}$ is a noncommutative nilpotent Lie group whose underlying manifold is $\mathbb{C}^{n} \times \mathbb{R}$ with coordinates $(z, t)=$ $\left(z_{1}, z_{2}, \ldots, z_{n}, t\right)$ and group law given by

$(z, t)\left(z^{\prime}, t^{\prime}\right)=\left(z+z^{\prime}, t+t^{\prime}+2 \Im m z . z^{\prime}\right)$ where $z . z^{\prime}=\sum_{j=1}^{n} z_{j} \bar{z}_{j} \quad z \in \mathbb{C}^{n}, \quad t \in \mathbb{R}$. 
Setting $z_{j}=x_{j}+y_{j}$, then $\left(x_{1}, x_{2}, \ldots, x_{n}, y_{1}, y_{2}, \cdots, y_{n}, t\right)$ forms a real coordinate system for $\mathbb{H}_{n}$. In this coordinate system, we define the following vector fields:

$$
X_{j}=\frac{\partial}{\partial x_{j}}+2 y_{j} \frac{\partial}{\partial t}, \quad Y_{j}=\frac{\partial}{\partial y_{j}}-2 x_{j} \frac{\partial}{\partial t}, \quad T=\frac{\partial}{\partial t} .
$$

It is clear from [7] that $\left\{X_{1}, X_{2}, \ldots, X_{n}, Y_{1}, Y_{2}, \ldots, Y_{n}, T\right\}$ is a basis for the left invariant vector fields on $\mathbb{H}_{n}$. These vector fields span the Lie algebra $\mathfrak{h}_{n}$ of $\mathbb{H}_{n}$ and the following commutation relations hold:

$$
\left[Y_{j}, X_{k}\right]=4 \delta_{j k} T, \quad\left[Y_{j}, Y_{k}\right]=\left[X_{j}, X_{k}\right]=\left[X_{j}, T\right]=\left[Y_{j}, T\right]=0 .
$$

Similarly, we obtain the complex vector fields by setting

$$
\left.\begin{array}{l}
Z_{j}=\frac{1}{2}\left(X_{j}-i Y_{j}\right)=\frac{\partial}{\partial z_{j}}+i \bar{z} \frac{\partial}{\partial t} \\
\bar{Z}_{j}=\frac{1}{2}\left(X_{j}+i Y_{j}\right)=\frac{\partial}{\partial \bar{z}_{j}}-i z \frac{\partial}{\partial t}
\end{array}\right\} .
$$

In the complex coordinate, we also have the commutation relations

$$
\left[Z_{j}, \bar{Z}_{k}\right]=-2 \delta_{j k} T, \quad\left[Z_{j}, Z_{j}\right]=\left[\bar{Z}_{k}, \bar{Z}_{k}\right]=\left[Z_{j}, T\right]=[\bar{Z}, T]=0 .
$$

The Haar measure on $\mathbb{H}_{n}$ is the Lebesgue measure $d z d \bar{z} d t$ on $\mathbb{C}^{n} \times \mathbb{R}[11]$. In particular, for $n=1$, we obtain the 3-dimensional Heisenberg group $\mathbb{H}_{1} \cong \mathbb{R}^{3}$ (since $\mathbb{C}^{n} \cong \mathbb{R}^{2 n}$ ). Hence $\mathbb{H}_{n}$ may also be referred to as $(2 n+1)$-dimensional Heisenberg group.

Let $K \subset G$ be a compact subgroup of $G$ and denote by $C_{c}(K \backslash G / K)$, the space of continuous functions with compact support on $G$ which satisfy $f\left(k_{1} g k_{2}\right)=f(g)$ for all $k_{1}, k_{2} \in K$. Such functions are called spherical functions or $K$-biinvariant functions. Then, $C_{c}(K \backslash G / K)$ forms a commutative Banach algebra under convolution.

Suppose that $K$ is a compact group acting by automorphism on $G$ via some homomorphism $\phi: K \rightarrow \operatorname{Aut}(G)$, we can form a semidirect product $K \ltimes G$ with the group law

$$
\left(k_{1}, n_{1}\right)\left(k_{2}, n_{2}\right)=\left(k_{1} k_{2}, n_{1}\left(k_{1} \cdot n_{2}\right)\right) \text {, where } k . n=\phi(k)(n) .
$$

Given a locally compact group $G$ and a compact subgroup $K \subset G$, the pair $(G, K)$ is called a Gelfand pair if $L^{1}(G / / K)$, the space of integrable, $K$ biinvariant functions on $G$ is commutative under convolution. Perhaps the best known examples are those defining symmetric spaces, that is a connected semisimple Lie group with finite centre and $K$ is maximal compact subgroup. The analysis associated with such pairs play an important role in the representation theory of semisimple Lie groups and has extensively been developed 
Helgason [9]. In sharp contrast to this, one might begin to assume that $G$ is a solvable Lie group. But then, if $G$ is simply connected (like $\mathbb{H}_{n}$ ) for example, there may be no non-trivial compact subgroups. One can however, consider pairs of the form $\left(K \ltimes H_{n}, K\right)$ where $K$ is a compact subgroup of $\operatorname{Aut}(G)$, the group of automorphism of $G$.

In the case, of $\mathbb{H}_{n}, \quad K \subseteq U(n)$ is a maximum compact connected subgroup of $\operatorname{Aut}\left(\mathbb{H}_{n}\right)$ for which $\left(K \ltimes H_{n}, K\right)$ is a Gelfand pair. This is denoted by $\left(K, \mathbb{H}_{n}\right)$. An important ingredient in this theory of Gelfand pair $\left(K, H_{n}\right)$ is the representation of $K$ on the space of polynomials $\mathscr{C}(V)$ given by $k . p(z):=p\left(k^{-1} z\right)$. The pair $\left(K \ltimes \mathbb{H}_{n}, K\right)$ is associated with the commutative Banach algebra $L^{1}(G / / K)$, whose Gelfand spectrum may be identified with the set of bounded spherical functions with compact open topology. Spherical functions are characterized as the joint eigenfunctions of all $G$-invariant differential operators on $G / K$, normalized in the $L^{\infty}$-norm [1]. More general theories on this can be seen for example in [2].

An elementary spherical function $\phi$ is defined to be a $K$-bi-invariant continuous function which satisfies $\phi(e)=1$ and such that $f \rightarrow f * \phi(e)$ defines an algebra homomorphism of $C_{c}(K \backslash G / K)$.

The elementary spherical functions are characterised by the following properties (see [9],[3]):

(i) They are eigenfunctions of the convolution operator:

$$
f * \phi=\hat{\phi}(f) \phi
$$

where

$$
\hat{\phi}(f)=\int_{G} f\left(x^{-1}\right) \phi(x) d x .
$$

(ii) They are eigenfunctions for a large class of left invariant differential operators on $G$.

(iii) They satisfy

$$
\int_{K} \phi(x k y) d k=\phi(x) \phi(y) .
$$

Now, on the Heisenberg group, we consider $K$, a compact subgroup of automorphisms of $\mathbb{H}_{n}$ such that the convolution algebra $L_{K}^{1}$ of $K$-invariant functions is commutative. A bounded, continuous $K$-invariant function $\varphi$ such that $f \rightarrow \int f \varphi$ is an algebra homomorphism on $L_{K}^{1}$ is called a $K$-spherical function. (For a complete characterisation of $K$-spherical functions and their properties (for various different $K$,) see [2],[3].) In fact, when $K=U(n)$, the $K$-spherical functions include the elementary spherical functions. 
Henceforth, let $G:=K \ltimes H_{n}$ be the semidirect product of $K$ and $\mathbb{H}_{n}$ via the group homomorphism $\phi: K \rightarrow \operatorname{Aut}\left(\mathbb{H}_{n}\right): \phi(k)(u)=k \cdot u=(k u, t)$. Here, $u=(z, t)=(x, y, t)$ where $z \in \mathbb{C}, t \in \mathbb{R}$ or $x, y \in \mathbb{R}^{n}, t \in \mathbb{R}$.

\section{Main Results}

Definition 2.1: A function $f: \mathbb{R}^{n} \longrightarrow \mathbb{R}$ is said to be radial if there is a function $\phi$ defined on $[0, \infty)$ such that $f(x)=\phi(|x|)$ for almost every $x \in \mathbb{R}^{n}$.

Simple and classical examples of radial functions and their properties can be seen in for example [9,p.266], [11,p.134] and [13,p.366].

Let $\rho$ be transformation on $\mathbb{R}^{n}$ and $x \in \mathbb{R}^{n}$. Then $\rho$ is said to be orthogonal if it is a linear operator on $\mathbb{R}^{n}$ that preserves the inner product $\langle\rho x, \rho y\rangle=\langle x, y\rangle$ for all $x, y \in \mathbb{R}^{n}$.

A Schwartz function $\varphi$ is said to be radial if for all orthogonal transformations, $A \in O(n)$ (i.e., for all rotations on $\mathbb{R}^{n}$ ), we have

$$
\varphi=\varphi \circ A \text {. }
$$

We shall denote the set of all radial Schwartz functions by $\mathrm{S}_{\text {rad }}\left(\mathbb{R}^{n}\right)$.

A distribution $u \in \mathrm{S}^{\prime}\left(\mathbb{R}^{n}\right)$ is called radial if for all orthogonal transformations $A \in O(n)$, we have

$$
u=u \circ A .
$$

This means

$$
\langle u, \varphi\rangle=\langle u, \varphi \circ A\rangle
$$

for all Schwartz functions $\varphi$ on $\mathbb{R}^{n}$. We denote by $\mathrm{S}_{\text {rad }}^{\prime}\left(\mathbb{R}^{n}\right)$ the space of all radial tempered distributions on $\mathbb{R}^{n}$.

Definition 2.2: A continuous function $\phi$ on a locally compact group $G$ is called positive definite if for each finite set $\left\{x_{1}, \cdots, x_{n}\right\}$ of $G$ and for all complex numbers $c_{1}, \cdots, c_{n}$,

$$
\sum_{i, j=1}^{n} c_{i} \overline{c_{j}} \phi\left(x_{i}^{-1} x_{j}\right) \geq 0
$$

or equivalently,

$$
\int_{G} \phi\left(x^{-1} y\right) f(x) \overline{f(y)} d x d y \geq 0
$$

for each $f \in C_{c}(G)$. If $\phi$ is positive definite, then

$$
|\phi(x)| \leq \phi(e), \quad \phi\left(x^{-1}\right)=\overline{\phi(x)}
$$


for all $x \in G$.

Definition 2.3: A distribution $T$ on $G$ is said is called positive definite if $T(\widetilde{\phi} * \phi) \geq 0$ for all $\phi$ in $\mathrm{D}(G)$. For $K$-bi-invariant distribution $T$ on $G$ to be positive definite, it is sufficient to verify $T(\widetilde{\phi} * \phi) \geq 0$ for all $\varphi \in C_{c}(K \backslash$ $G / K)$. However, this is not generally true except for tempered $K$-bi-invariant distributions.

In what follows, we shall raise the new radial distribution generated from the Heisenberg heat equation and show that it satisfies the conditions in [1]. To do this, recall that the Heisenberg heat equation is defined on $\mathbb{H}_{n} \times \mathbb{R}^{+}$by

$$
\partial_{t} U(h, t)=\Delta U(h, t), \quad U(h, t) \in \mathbb{H}_{n} \times \mathbb{R}^{+} .
$$

The fundamental solution of this equation is given by the heat kernel $K_{t}(h)$ which is obtained explicitly in [10] as

$$
K_{t}(x, u, \xi)=c_{n} \int_{\mathbb{R}} e^{-i \lambda E} e^{-t \lambda^{2}}\left(\frac{\lambda}{\sin h \lambda t}\right)^{n} e^{-\frac{1}{4} \lambda(\cot h t \lambda)(x \cdot x+u \cdot u)} d \lambda
$$

where $c_{n}=(4 \pi)^{-n}, \quad \lambda \in \mathbb{R}^{*}=\mathbb{R} \backslash\{0\}$.

Let $\varphi_{\lambda}^{k}$ be the K-spherical function on $\mathbb{H}_{n}$. That is the distinguished spherical function restricted to $L^{1}(K \backslash G / K)$ where $(K, G)$ is a Gelfand pair, $K$ a compact subgroup of $A u t\left(\mathbb{H}_{n}\right)$. In this case, $G$ may be taken as a semi-direct product of $K$ and $\mathbb{H}_{n}$ (i.e., $\left.G:=K \ltimes \mathbb{H}_{n}\right)[2]$. Thus $\varphi_{\lambda}^{k}$ is a unique radial function since it is a radial eigenfunction of the Heisenberg Laplacian [11,p.38]. (In fact, elementary spherical functions are radial functions [13].) i.e.

$$
\varphi_{\lambda}^{k}(u)=\psi(|u|)
$$

Now rewriting the heat kernel, we have

$$
\begin{aligned}
\Phi_{t}(h) & =c_{n} \int_{\mathbb{R}^{n}} \mathrm{e}^{-\lambda \xi} \mathrm{e}^{-t \lambda^{2}} \varphi^{n}(\lambda t) \mathrm{e}^{-\frac{1}{4}|h|^{2} \phi(\lambda t)} d \lambda \\
& =c_{n} \int_{\mathbb{R}^{n}}^{\lambda\left(\xi+\lambda^{2}\right)} \varphi^{n}(\lambda t) \mathrm{e}^{-\frac{1}{4}|h|^{2} \phi(\lambda t)} d \lambda \\
& =c_{n} \psi_{\lambda}(|h|, t)
\end{aligned}
$$

which gives a radial function for $K:=U(n)$ and

$$
\Phi_{t}(h)=c_{n} \psi_{\lambda}\left(\mathrm{e}^{-i \theta}|h|, t\right)
$$


which gives a polyradial function for $K:=\mathbb{T}^{n}$. Applying dilations to the radial function, we obtain

$$
\begin{aligned}
\Phi_{t}(h) & =\delta_{r}\left(c_{n} \psi_{\lambda}(|h|, t)\right. \\
& =c_{n} \psi_{\lambda}\left(r|h|, r^{2} t\right) \\
& =c_{n} t^{-n / 2} \varphi^{n}(h) \delta_{r}^{-2}(h) \mathrm{e}^{-|h|^{2} / 4 t}
\end{aligned}
$$

Notice that $\Phi_{t}(h)$ as defined in (2.1) is homogeneous and locally integrable, thus a regular distribution on $\mathbb{H}_{n}$. In fact, it is bounded and its Fourier transform exists and is radial [5]. Also, $\Phi_{t}(h)$ is compactly supported and is in $\mathrm{S}\left(\mathbb{C}^{n}\right)$. Thus, its Fourier transform $\mathcal{F} \Phi_{t}$ is also in $\mathrm{S}\left(\mathbb{R}^{n}\right)$ and $\int \Phi_{t}(h) d h=1$.

Next, for any $u \in \mathrm{S}^{\prime}\left(\mathbb{H}_{n}\right)$,

$$
\left\langle u^{r a d}, \Phi_{t}(h)\right\rangle=\left\langle u, \Phi_{t}(h)^{r a d}\right\rangle
$$

holds since $\Phi_{t}$ is radial[1].

We therefore seek a condition for positive definiteness of this radial function on the Heisenberg group as defined in (2.1). The restrictive conditions for positive definiteness of radial functions with compact support can be seen in [14].

Proposition: 2.4 Let $\Phi_{t}(h)$ be as given in (2.1). Assume that $\varphi_{\lambda}^{k}(h)$ is square integrable and continuous. Then $\Phi_{t}(h)$ is positive definite on $\mathbb{R}^{n}$ if, and only if, for every $\lambda \in \mathbb{R}^{*}$, the map $\varphi_{\phi(\lambda)}^{n}(t)=\varphi^{n}(\lambda t) \phi(\lambda t)$ is positive definite on $\mathbb{R}^{n}$.

Proof: Clearly, $\varphi_{\lambda}^{k}$ is square integrable [1]. If $\varphi_{\phi(\lambda)}^{n}(t)$ is positive definite for every $t$, then $\Phi_{t}(h)$ is positive definite since $|h|$ is even.

Conversely, if $\Phi_{t}(h)$ is positive definite, then $\varphi_{\phi(\lambda)}^{n}(t)$ is positive definite since $\mathrm{e}^{-|h|^{2} / 4 t}$ is always positive. Thus, $\Phi_{t}(h)$ is a Fourier transform of some positive Borel measure on $\mathbb{R}^{3}$. Thus, $\varphi^{n}(\lambda t) \phi(\lambda t)$ is positive definite by corollary 6 of [6].

The next theorem follows from Astengo and Ricci [1].

Theorem: $2.5 \Phi_{t}(h)$ is compactly supported in $\mathrm{S}^{\prime}\left(\mathbb{H}_{n}\right)$.

Proof: First, notice that $\Phi_{t}(h)$ is a radial distribution on $\mathbb{H}_{n}$ by definition. Thus, the Gelfand transform, $\mathcal{G} \Phi_{t}(h)$ exists and is bounded. By proposition 5.2 of $[1], \Phi_{t}(h)$ is compactly supported and $\mathcal{G} \Phi_{t}(h)=\widehat{\Phi_{t}}$. Now, for any $\psi \in$ 
$\mathrm{S}\left(\mathbb{R}^{2}\right)$, the integral $\int_{\sum} \widehat{\Phi_{t}} \psi d \mu$ is absolutely convergent and therefore, $\widehat{\Phi_{t}}$ is in $\mathrm{S}^{\prime}\left(\mathbb{H}_{n}\right)^{\mathrm{rad}}$. In fact,

$$
\left|\widehat{\Phi_{t}}(h)\right|=\left|\left\langle\Phi_{t}, \varphi_{k}^{\lambda}\right\rangle_{H_{n}}\right| \leq C\left\|\varphi_{k}^{\lambda}\right\|_{C^{m}(K)}
$$

where $K=\operatorname{supp} \Phi \subset \sum$. By [1, Lemme 3.2], $\Phi_{t}$ is rapidly decreasing on $\sum$ and so, for every $\psi \in \mathrm{S}_{\text {rad }}\left(\mathbb{R}^{2}\right)$, the integral $\int_{\sum} \widehat{\Phi_{t}} \psi d \mu$ is absolutely convergent. Therefore, the conditions of $\left[1\right.$, Prop.5.2.] is satisfied and thus $\Phi_{t}$ is compactly supported.

\section{References}

[1] Astengo, B., Blasio D.B. and Ricci F.: Paley-Wierner Theorems for the $U(n)$-spherical transform on the Heisenberg group, arXiv:1303.0997v1 [math.FA] 5 Mar 2013.

[2] Astengo, B., Blasio D.B. and Ricci F.: Gelfand pairs on the Heisenberg group and Schwartz functions. J. Funct. Anal. 256(2009.) (5), 15651587.

[3] Benson, C., Jenkins, J. and Ratcliff, G.: Bounded K-Spherical functions on the Heisenberg groups. J. Funct. Anal., 105 (1992), 409-443.

[4] Benson C., Jenkins J., Ratcliff G., The spherical transform of a Schwartz functions on the Heisenberg group. J. Funct. Anal., 154(1998), 379-423.

[5] Bochner, S. and Chandrasekharan, K.: Fourier transforms. Princeton Univ. Press, London, 1949.

[6] Fischer D.R.: Functions Positive-Definite on $R^{3}$ and the Heisenberg Group, J. Funct. Anal., 42(1981), 338-346.

[7] Folland, G.B and Stein, E.M.: Estimates for the $\bar{\partial}_{b}$ complex and Analysis on the Heisenberg Group, Communications on Pure and Applied Maths. Vol XXVII (1974) 429-522.

[8] Gangolli, R.: Spherical functions on semisimple Lie groups, in symmetric spaces edited by W. Boothy and G. Weiss, Marcel Dekker, Inc. N.Y., 1972.

[9] Helgason, S.: Groups and geometric analysis: Integral geometry, differential operators and spherical functions. Academic Press Inc., New York, 1984 
[10] Krotz, B., Thangavelu, S. and Xu, Y.: The heat Kernel transform for the Heisenberg group. arXiv:math.CA/0401243v2, 2005.

[11] Mauceri, G.: Zonal Multipliers On the Heisenberg group, Pacific J. Math. 95(1981), 143-169.

[12] Stein, E.M. and Weiss, G.: Introduction to Fourier analysis on Euclidean spaces. Princeton, Univ. Press, London, 1971.

[13] Szego G., Orthogonal polynomials. AMS Colloq. Publ., Vol. XXIII, AMS Providence, Rhode Island, 1939.

[14] Zongmin Wu: Compactly supported positive definite radial functions, Adv. in Comput. Math. 4(1995), 283-292.

Received: March 1, 2014 